\title{
Enhancing High-Quality Data Management Services in Non-commercial
}

Clinical Trials

Christian Ohmann, Steve Canham, Serena Battaglia, Jacques Demotes, Christine Toneatti

The ECRIN certification programme ${ }^{1}$ is designed to audit data centres to confirm their ability to provide compliant effective and efficient data management services for clinical trials as well as to provide a clear interpretation of regulatory and good practice requirements.

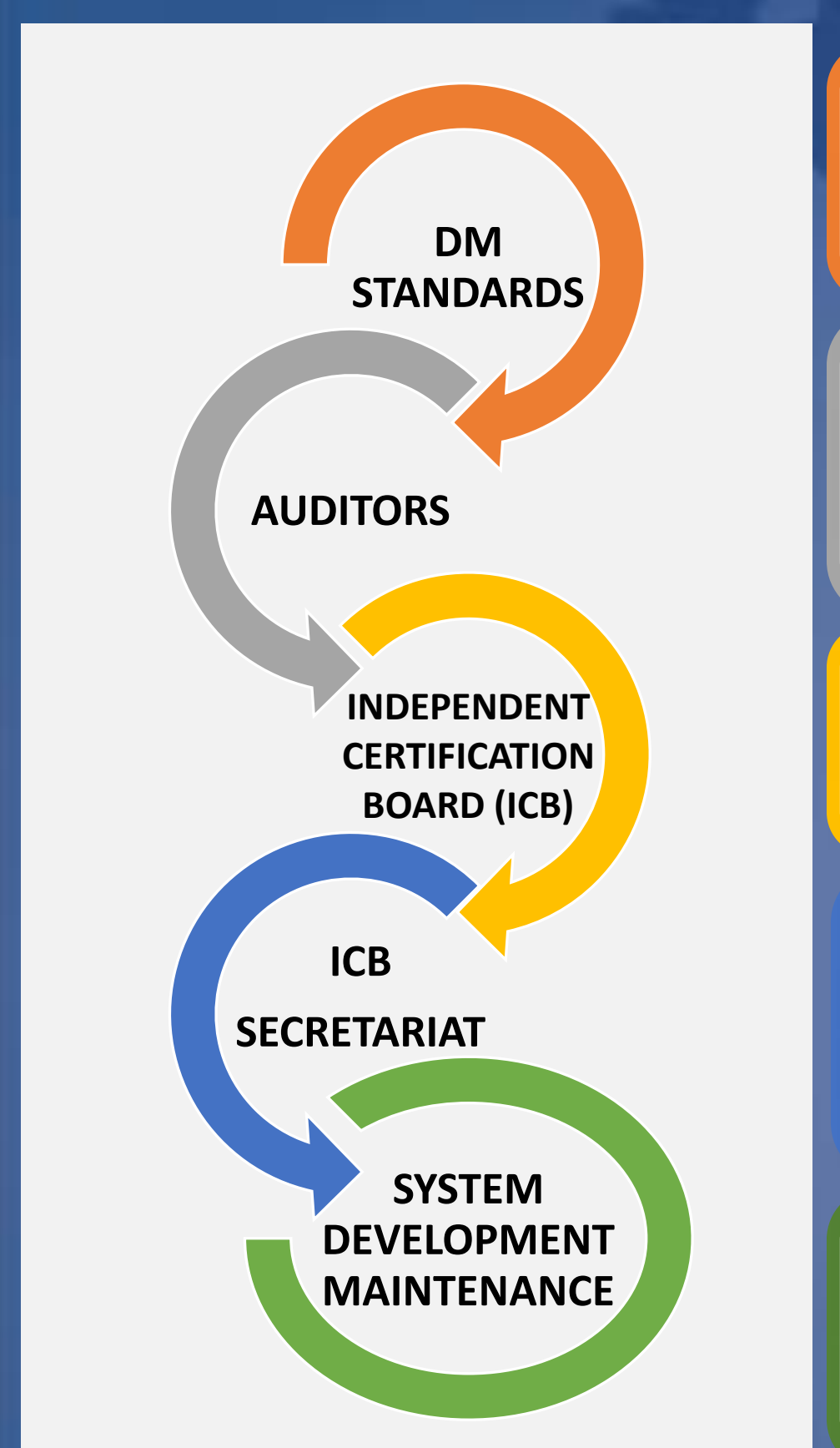

106 standards split in 3 categories: General, IT and Data Management ${ }^{2}$. Developed by an ECRIN expert group.

14 qualified IT/DM/Quality auditors. Perform independent audits of applicant data centres for certification, (Readiness, Initial, CAPA review, re-audit, certification renewal audits).

Objective and independent assessment of the audit results to decide for certification.

Managing the certification programme (annual calls, set up and support of ICB, audit planning, performance and reporting, maintaining auditors' expertise and capacity).

Development and maintenance of programme procedures, and supporting tools for audit observations tracking, grading, and reporting.

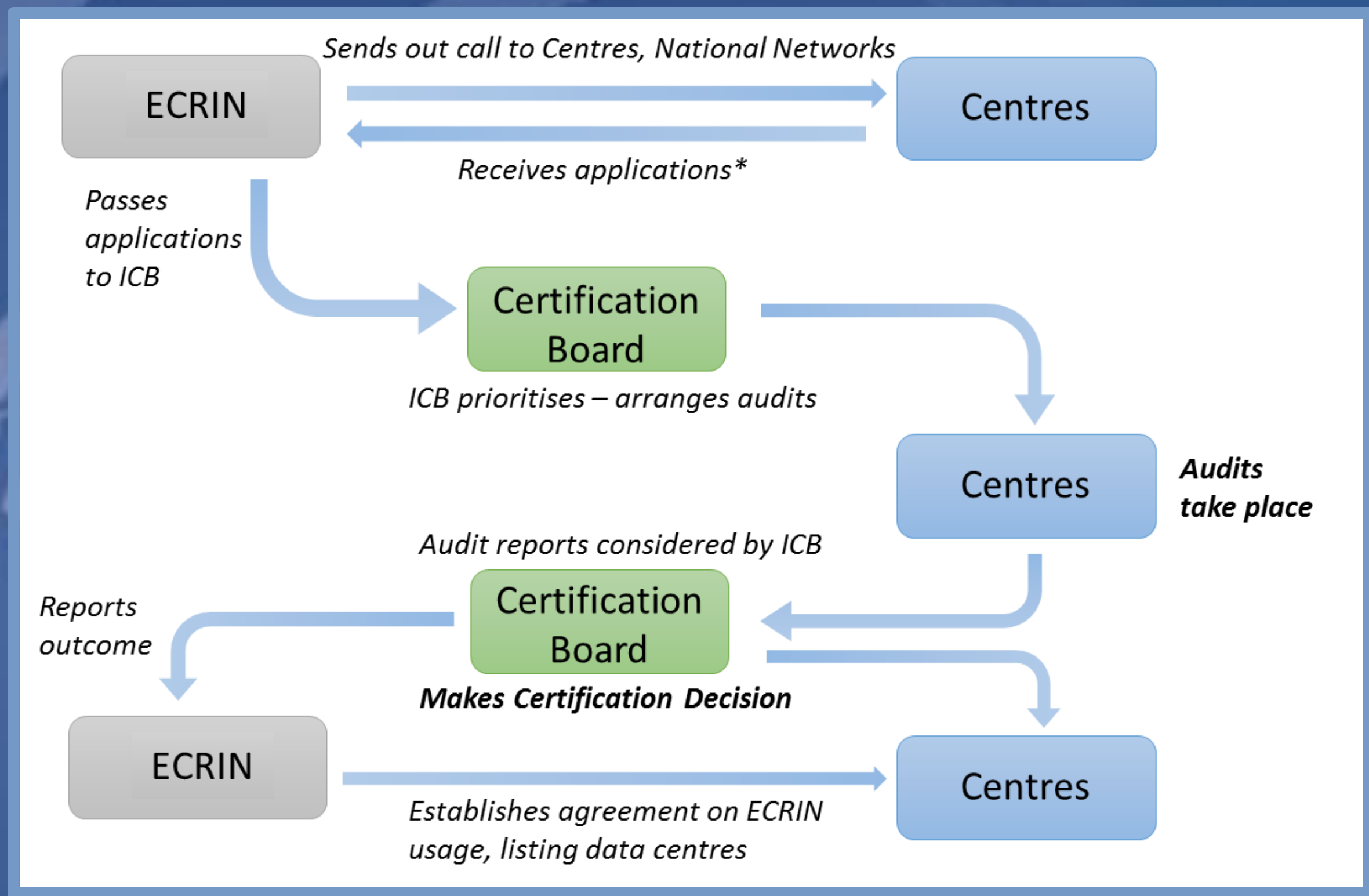

The ECRIN Data Centre Certification programme supports and supervises the implementation of high-quality clinical data management to promote further harmonisation and standardisation of data management practice worldwide.
13 certified centres in EU 1 in Japan $(2015-2019)^{3}$

Ongoing certification of 1 centre in Korea
Expanding the model beyond Europe: Collaboration with Eastern countries (Singapore, Japan, South Korea and Taiwan); - Data Management standards translated in Japanese, Korean, Chinese;

- Auditor trainings (theoretical and on-the-job training)

${ }^{1}$ Ohmann et al., Contemp Clin Trials Commun. 2017 Mar; 5: 153

${ }^{2}$ https://zenodo.org/record/1240941\#.XkPBzyPjKUk

${ }^{3}$ https://www.ecrin.org/who-we-are/partners 